\title{
miR-124 regulates the osteogenic differentiation of bone marrow-derived mesenchymal stem cells by targeting Sp7
}

\author{
JIA-ZHEN TANG ${ }^{1 *}$, XIAO LIN ${ }^{2 *}$, JIA-YU ZHONG ${ }^{2}$, FENG XU ${ }^{3}$, FENG WU ${ }^{4}$, \\ XIAO-BO LIAO $^{5}$, RONG-RONG CUI ${ }^{3}$, FUXINGZI LI ${ }^{3}$ and LING-QING YUAN ${ }^{3}$ \\ ${ }^{1}$ Department of Endocrinology and Metabolism, The First Affiliated Hospital of Nanchang University, Nanchang, \\ Jiangxi 330031; ${ }^{2}$ Department of Geriatrics, Institute of Aging and Geriatrics, The Second Xiang-Ya Hospital, \\ Central South University; ${ }^{3}$ Department of Endocrinology and Metabolism, \\ National Clinical Research Center for Metabolic Diseases, The Second Xiang-Ya Hospital, \\ Central South University; Departments of ${ }^{4}$ Pathology and ${ }^{5}$ Cardiovascular Surgery, The Second \\ Xiang-Ya Hospital, Central South University, Changsha, Hunan 410011, P.R. China
}

Received July 20, 2018; Accepted March 6, 2019

DOI: $10.3892 / \mathrm{mmr} .2019 .10054$

\begin{abstract}
MicroRNAs (miRNAs) are novel key regulators of cellular differentiation. miR-124 has been reported to regulate osteogenic differentiation of bone marrow-derived mesenchymal stem cells (BMSCs). However, the specific mechanisms involved have not yet been fully elucidated. The present study aimed to investigate the effect of miR-124 on osteogenic differentiation of BMSCs and its underlying mechanisms. In the present study, it was found that alkaline phosphatase (ALP) activity, osteocalcin (OC) secretion, and the protein levels of osterix (Sp7) and runt-related transcription factor 2 (Runx2) were significantly increased, whereas the expression of miR-124 was decreased in a time-dependent manner during osteogenic differentiation of BMSCs. Following overexpression of miR-124 via transfection of miR-124 mimics in BMSCs, Runx2 protein expression and ALP activity were significantly decreased. By contrast, inhibition of miR-124 expression led to an increase in ALP activity and Runx2 expression. Sp7 expression was suppressed in BMSCs transfected with miR-124 mimics while increased when miR-124 expression was inhibited, indicating that miR-124 regulates the expression of Sp7. Moreover, a luciferase reporter assay further verified that $\mathrm{Sp} 7$
\end{abstract}

Correspondence to: Professor Ling-Qing Yuan or Dr Fuxingzi Li, Department of Endocrinology and Metabolism, Hunan Provincial Key Laboratory of Metabolic Bone Diseases, National Clinical Research Center for Metabolic Diseases, The Second Xiang-Ya Hospital, Central South University, 139 Middle Renmin Road, Changsha, Hunan 410011, P.R. China

E-mail: allenylq@csu.edu.cn

E-mail:1fxz9394@csu.edu.cn

*Contributed equally

Key words: miR-124, bone marrow-derived mesenchymal stem cells, osteogenic differentiation, $\mathrm{Sp} 7$ is the direct target of miR-124. Finally, the effect of miR-124 inhibitor on promoting the differentiation of BMSCs was abolished following treatment with a small interfering RNA targeting Sp7. Taken together, the present study demonstrates that miR-124 inhibits the osteogenic differentiation of BMSCs by targeting Sp7.

\section{Introduction}

Osteoporosis is becoming a serious public health issue as aging of the global population accelerates. Bone marrow-derived mesenchymal stem cells (BMSCs) are multipotent progenitor cells with the capacity of differentiating into osteoblasts, adipocytes, chondrocytes, myoblasts and neurocytes $(1,2)$. The differentiation of osteoblasts and adipocytes from BMSCs, as well as the balance between osteoclastic bone resorption and osteoblastic bone formation, contribute to the maintenance of bone volume (3). With increasing age, the imbalance between osteogenesis and adipogenesis of BMSCs results in a decrease in bone mineral density leading to osteoporosis (4). Therefore, the balance between bone marrow osteogenesis and adipogenesis may represent another therapeutic target for the prevention or treatment of osteoporosis. However, the mechanisms regulating the osteogenesis of BMSCs require further exploration.

miRNAs are a series of 20-24 nt non-coding RNAs that act as epigenetic negative regulators of their target mRNAs by directly binding to the $3^{\prime}$-untranslated region (3'UTR) post-transcriptionally $(5,6)$. Recently, miRNAs have been shown to play a key role in bone homeostasis and to regulate a variety of cellular functions, including differentiation, proliferation and migration (7-9). For example, miR-133 directly targets Runx2 and regulates BMP-2-mediated osteogenic differentiation of $\mathrm{C} 2 \mathrm{C} 12$ cells (10) and $\beta$-glycerophosphate ( $\beta$-GP)-induced vascular smooth muscle cell (VSMC) osteoblastic differentiation (7). In addition, miR-125b has been shown to suppress osteogenic differentiation of BMSCs via targeting Sp7 (11). miR-124 also plays an important role 
in the differentiation of BMSCs, including myogenic (12), neuronal (13), and osteogenic differentiation (14). However, the mechanisms involved in miR-124-mediated osteogenic differentiation of BMSCs require elucidation.

In the present study, the miR-124 regulation of osteogenic differentiation of BMSCs was investigated and it was revealed that miR-124 regulated the osteogenic differentiation of BMSCs by targeting Sp7.

\section{Materials and methods}

Culture of BMSCs and cell transfection. Human BMSCs were isolated from bone marrow samples as described in our previous study (15). The specimens were from three subjects [aged 24 (female), 26 (male) and 30 years (male)] undergoing routine therapeutic surgery at the Department of Orthopaedic Surgery from December 2016 to March 2017, The Second Xiang-Ya Hospital, Central South University (Changsha, China). All of the subjects provided informed consent. Approval for the present study was acquired from the Medical Ethics Committee of The Second Xiang-Ya Hospital, Central South University. The bone marrow samples were harvested using standard Ficoll-Hypaque density sedimentation methods. BMSCs were selected based on adhesion and proliferation on a tissue culture plastic substrate. Cells from passages 3-5 were used in the experiments.

For cell transfection, miR-124 mimics, miR-124 inhibitor, and the negative controls, or Sp7 small interfering RNA (siRNA) oligos (RiboBio, Guangzhou, China) were transfected into BMSCs using Lipofectamine 2000 (Invitrogen; Thermo Fisher Scientific, Inc., Waltham, MA, USA) according to the manufacturer's instructions. After $6 \mathrm{~h}$ of co-culture, the cells were cultured in Dulbecco's modified Eagle's medium (DMEM) supplemented with $10 \%$ foetal bovine serum (FBS; both Gibco; Thermo Fisher Scientific, Inc.) and cultured for a further 24-48 h.

Osteogenic differentiation. To induce osteogenic differentiation, BMSCs were cultured in osteogenic medium (basal medium $+50 \mu \mathrm{g} / \mathrm{ml}$ phospho-ascorbate $+10 \mathrm{mM} \beta-\mathrm{GP}$ ) for 3-7 days. To assess osteogenic differentiation, an ALP activity assay (16) was performed using the spectrophotometric measurement of $p$-nitrophenol release at $37^{\circ} \mathrm{C}$.

Alizarin Red S staining. Alizarin Red S staining was performed as previously described (17). Briefly, BMSCs cultured in osteogenic medium for 14 days were fixed in $4 \%$ paraformaldehyde for $30 \mathrm{~min}$ at room temperature and stained with $2 \%$ ( $\mathrm{pH} 4.2$ ) Alizarin Red S solution for 1-3 min at $37^{\circ} \mathrm{C}$. Next, cells were washed with phosphate-buffered saline (PBS) three times to eliminate non-specific staining. The stained matrixes were assessed and photographed using a digital microscope. To quantify calcium levels, cells were washed with PBS and decalcified with $0.6 \mathrm{~N} \mathrm{HCl}$ for $24 \mathrm{~h}$. Calcium content was determined by measuring the concentration of calcium in the $\mathrm{HCl}$ supernatant by atomic absorption spectroscopy. Following decalcification, the cells were washed with PBS three times and solubilized with $0.1 \mathrm{~N}$ $\mathrm{NaOH} / 0.1 \%$ SDS. The protein content was measured with a BCA protein assay (Beyotime Biotechnology, Shanghai,
China). The calcium content of the cell layer was normalized to the protein content.

Western blotting. To investigate the protein levels of Runx2 and $\mathrm{Sp} 7$, western blotting was performed as previously described $(16,18)$. Total protein was extracted from BMSCs using radioimmunoprecipitation assay lysis buffer (Beijing Solarbio Science \& Technology Co., Ltd., Beijing, China) and the concentration of protein was determined a Bicinchoninic Acid protein kit. A total of $30 \mu \mathrm{g}$ protein was loaded per lane in $10 \%$ electrophoresis gel and separated with sodium dodecyl sulphate-PAGE and transferred to polyvinylidene fluoride membranes (Millipore, Billerica, MA, USA). Membranes were blocked with 5\% non-fat milk in Tris-buffered saline for at least $1 \mathrm{~h}$ at room temperature. The membranes were incubated with primary antibodies, including anti-Runx2 (cat. no. ab76956; 1:1,000), anti-Sp7 (cat. no. ab209484, 1:1,000; both Abcam, Cambridge, UK), and anti- $\beta$-actin (cat. no. AM1021B, 1:3,000; Abgent, Inc., San Diego, CA, USA) at $4^{\circ} \mathrm{C}$ overnight, followed by incubation with the horseradish peroxidase-conjugated goat anti-rabbit (cat. no. sc-2004, 1:5,000) or horseradish peroxidase-conjugated goat anti-mouse (cat. no. sc-2005, 1:5,000; both Santa Cruz Biotechnology, Santa Cruz, CA, USA) secondary antibodies at $37^{\circ} \mathrm{C}$ for $1 \mathrm{~h}$. The immunoreactive bands were visualised using the enhanced chemiluminescence Plus Western blot detection kit (Amersham Biosciences UK Ltd., Little Chalfont, Buckinghamshire, UK) and densitometric quantification of band intensity from three independent experiments was carried out with Image-Pro Plus 6.0 software (Media Cybernetics, Inc., Rockville, MD, USA). The relative expression levels of target proteins were normalized to the intensity of the $\beta$-actin band.

$R T$-qPCR analysis. Total small RNA was extracted using the mirVana miRNA Isolation Kit (Ambion, Austin, TX, USA). The cDNA was synthesised via an All-in-One ${ }^{\mathrm{TM}}$ miRNA first-strand cDNA synthesis kit (AMRT-0060; GeneCopoeia, Inc., Rockville, MD, USA). Briefly, a $25 \mu 1$ reverse transcription reaction was carried out for $60 \mathrm{~min}$ at $37^{\circ} \mathrm{C}, 5 \mathrm{~min}$ at $85^{\circ} \mathrm{C}$, and then held at $4^{\circ} \mathrm{C}$. To analyse the expression of miR-124, RT-qPCR was performed with All-in-One ${ }^{\mathrm{TM}} \mathrm{qPCR}$ Mix (QP003; GeneCopoeia, Inc.) using the LightCycler ${ }^{\circledR} 96$ System (Roche Diagnostics, Indianapolis, IN, USA). For qPCR analysis, $20 \mu 1$ reactions were incubated in a 96-well optical plate at $95^{\circ} \mathrm{C}$ for $10 \mathrm{~min}$, followed by 40 cycles of $95^{\circ} \mathrm{C}$ for $10 \mathrm{sec}, 62^{\circ} \mathrm{C}$ for $20 \mathrm{sec}$, and $72^{\circ} \mathrm{C}$ for $20 \mathrm{sec}$. U6 snRNA was used as the reference. The miR-124 (HmiRQP0074) and U6 (HmiRQP9001) primers were purchased from GeneCopoeia. The relative standard curve method $\left(2^{-\Delta \Delta C q}\right)$ was used to determine the relative mRNA and miRNA expression (19). Results are expressed as fold changes relative to the relevant control. qPCR was performed in triplicate and the results are presented as the mean \pm standard error of samples.

Luciferase reporter assay. A segment of the Sp7 3-untranslated region (3'UTR) containing the predicted miR-124 binding sites was amplified by PCR. The PCR products were purified and inserted into the XbaI-FseI site downstream of the stop codon in the pGL3 control luciferase reporter vector (Promega Corporation, Madison, WI, USA), resulting in WT-pGL3-Sp7. 
To construct MUT-pGL3-Sp7, the Quick Change Site-Directed Mutagenesis kit (Stratagene; Agilent Technologies, Inc., Santa Clara, CA, USA) was used to induce point mutations in the 3'UTR of WT-pGL3-Sp7. The primer sequences for the PCR are shown in Table I. BMSCs were cotransfected with the luciferase reporter carrying WT-pGL3-Sp7 or MUT-pGL3-Sp7 and miR-124 mimics or scramble control oligos. After 48 h, luciferase activity was detected with the luciferase assay system (Promega Corporation).

Statistical analysis. The results are presented as means \pm standard deviation. Analysis was performed with SPSS software (version 17.0; SPSS Inc., Chicago, IL, USA). For comparisons of two groups, the Student's t-test was used, and comparisons between values of more than two groups were evaluated by one-way analysis of variance (ANOVA) together with a Tukey's post hoc test. A level of $\mathrm{P}<0.05$ was considered statistically significant. Representative figures are shown in the study.

\section{Results}

Expression of miR-124 is decreased during osteogenic differentiation of BMSCs. To examine the potential osteogenic differentiation ability of BMSCs, human BMSCs were cultured in osteogenic medium. The osteoblastic differentiation markers ALP activity, OC secretion, and osterix and Runx2 expression $(20,21)$ were assessed. As expected, ALP activity and OC secretion, as well as the protein expression of osterix and Runx2, were significantly increased (Fig. 1A-D). In addition, Alizarin Red S staining verified that mineralization nodules were formed in BMSCs cultured in osteogenic medium for 14 days (Fig. 1E). However, RT-qPCR showed that the expression of miR-124 was considerably decreased in a time-dependent manner during osteogenic differentiation of BMSCs (Fig. 1F). These results showed that BMSCs cultured in osteogenic medium could differentiate into osteoblasts and that miR-124 may be involved in osteogenic differentiation of BMSCs.

miR-124 inhibits osteogenic differentiation of BMSCs. To investigate whether miR-124 exerts a regulatory effect on osteogenic differentiation of BMSCs, a gain- and loss-of-function approach was used to overexpress or inhibit the expression of miR-124, respectively. RT-qPCR verified that miR-124 mimics induced miR-124 expression by more than 70-fold whereas the miR-124 inhibitor significantly decreased the expression of miR-124 (Fig. 2A). In addition, ALP activity and $\mathrm{OC}$ and Runx 2 protein expression were significantly decreased in miR-124-overexpressing cells while inhibition of miR-124 expression led to opposite results (Fig. 2B-D). Taken together, our results demonstrated that miR-124 could regulate osteogenic differentiation of BMSCs.

Sp7 is the target gene of miR-124. To investigate the mechanism underlying miR-124 regulation of osteogenic differentiation of BMSCs, we searched for potential targets of miR-124 using miRNA target prediction algorithms and identified $\mathrm{Sp} 7$ as a putative target gene of miR-124 (Fig. 3A). To confirm this target, a luciferase reporter assay was performed
Table I. Nucleotide sequences of primers for WT and mutant reporter plasmids.

\begin{tabular}{ll}
\hline Gene & \multicolumn{1}{c}{ Primer sequence (5' to 3') } \\
\hline WT-Sp7 & F: 5'-GCTCTAGAGCCCATCTCCACCTCCA \\
& GT-3' \\
& R: 5'-GGCCGGCCATCCCTTCCATTCCAC \\
& AA-3' \\
MUT-Sp7 & F: 5'-AAGCCGCTTCGTGTCTATCCTGTAA \\
& CGTTGGA-3 \\
& R: 5'-TTCGGCGAAGCACAGATAGGACAT \\
& TGCAACCT-3
\end{tabular}

WT, wild-type; MUT, mutant, F, forward; R, reverse.

to evaluate whether miR-124 could directly target the 3'UTR of Sp7. Luciferase reporters containing the wild-type (WT) or mutant (MUT) 3'UTR uncoding sequences for the predicted miRNA binding sites of Sp7 were introduced with miR-124 mimics into BMSCs. The results showed that overexpression of miR-124 decreased the luciferase activity of the WT-Sp7 3'UTR reporter genes while mutation of $\mathrm{Sp} 7$ binding sites abolished this repression. These results suggested that $\mathrm{Sp} 7$ is a direct target of miR-124 (Fig. 3B). Moreover, overexpression of miR-124 in BMSCs resulted in decreased expression of Sp7, whereas inhibition of miR-124 expression upregulated the expression of $\mathrm{Sp} 7$ (Fig. 3C). These results provide evidence that miR-124 directly targets $\mathrm{Sp} 7$.

Sp7 is involved in the miR-124 regulation of osteogenic differentiation of BMSCs. To further confirm that miR-124 relies on $\mathrm{Sp} 7$ to regulate osteogenic differentiation of BMSCs, the effect of Sp7 siRNA treatment on osteoblastic markers involved in the osteogenic differentiation of BMSCs was determined. As shown in Fig. 4A, Sp7 was successfully knocked down by Sp7-targeting siRNA. Transfection with Sp7 siRNA significantly reduced ALP activity and the protein levels of Runx2, whereas transfection with the miR-124 inhibitor significantly increased ALP activity and Runx2 expression. Nevertheless, Sp7 siRNA completely blocked the ALP activity induced by miR-124 inhibitors (Fig. 4B and C). These data confirmed that miR-124-dependent inhibition of ALP activity was dependent on Sp7.

\section{Discussion}

In the present study, it was demonstrated that miR-124 was downregulated during osteogenic differentiation of bone marrow-derived mesenchymal stem cells (BMSCs) and involved in regulating the osteogenic differentiation of BMSCs. In addition, our results confirmed that $\mathrm{Sp} 7$ is a direct target of miR-124 and is also involved in regulating the osteogenic differentiation of BMSCs. Taken together, our results showed that miR-124 negatively regulates osteogenic differentiation of BMSCs by targeting Sp7.

MSCs are commonly isolated from adult bone marrow and have the potential to differentiate into adipose tissue, bone, 
A

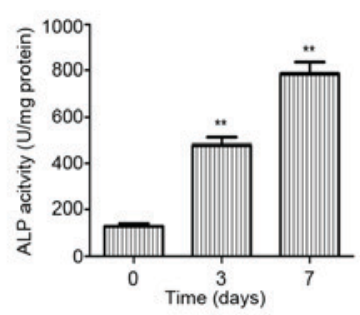

B

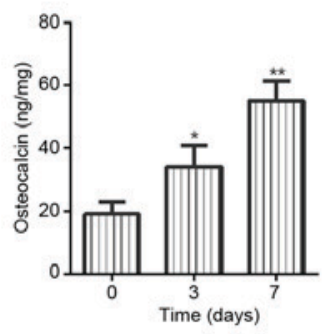

E

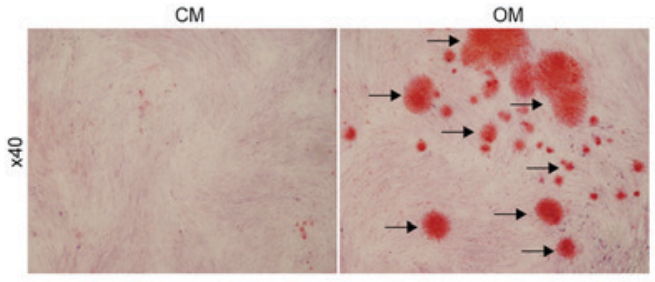

C

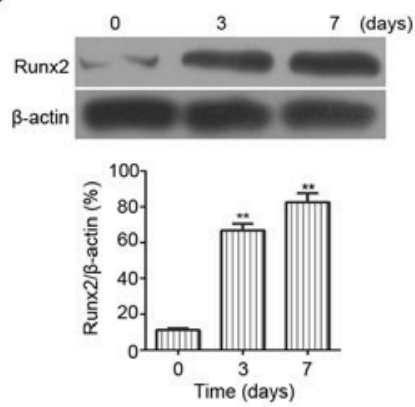

F

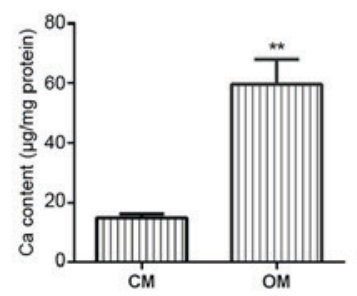

D
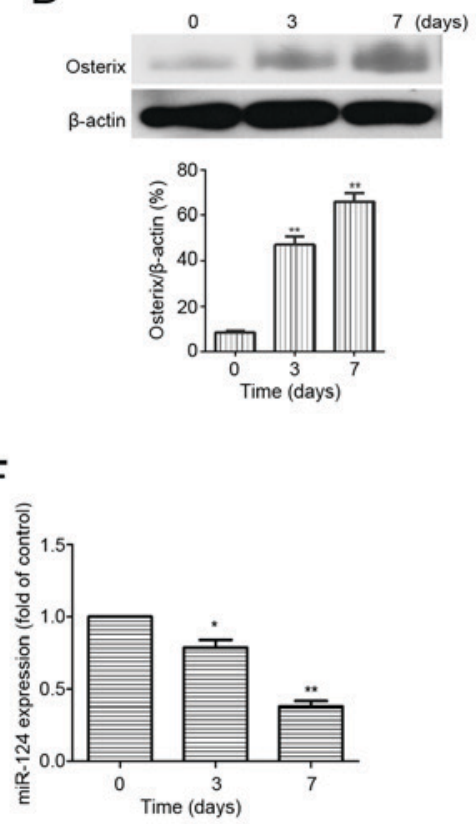

Figure 1. Expression of miR-124 during osteogenic differentiation of bone BMSCs. (A) ALP activity was determined using spectrophotometric methods during osteogenic differentiation of BMSCs. (B) OC secretion was measured by ELISA. (C and D) Western blotting was performed to determine the expression of Runx2 and osterix during osteogenic differentiation of BMSCs. (E) Alizarin Red S staining showed the formation of mineralization nodules in BMSCs cultured in CM and OM for 14 days, respectively. The black arrow indicates the mineralization nodules. (F) The expression of miR-124 was detected by RT-qPCR during osteogenic differentiation of BMSCs. Data are expressed as means \pm standard deviation (SD), $n=3$. " $\mathrm{P}<0.05,{ }^{* * *} \mathrm{P}<0.01$ compared with the control group. BMSCs, bone marrow-derived mesenchymal stem cells; ALP, alkaline phosphatase; OC, osteocalcin; CM, control medium; OM, osteogenic medium.

A

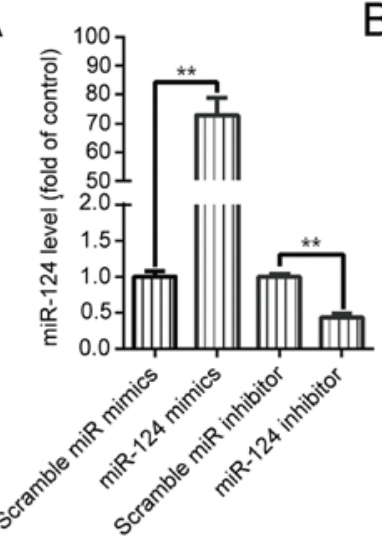

D

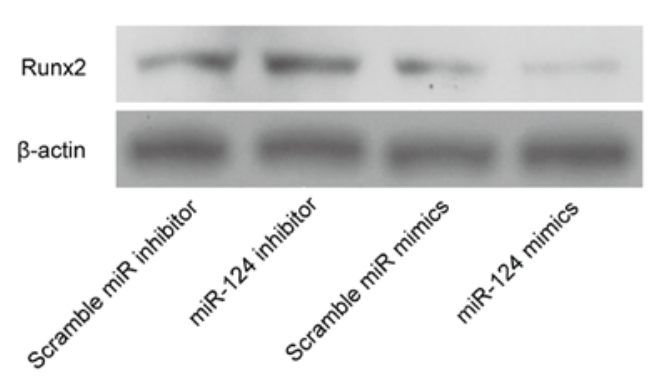

B

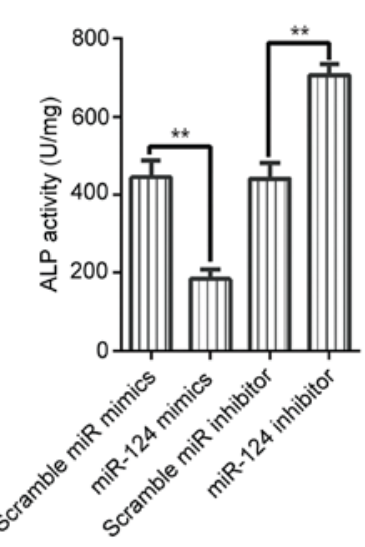

C

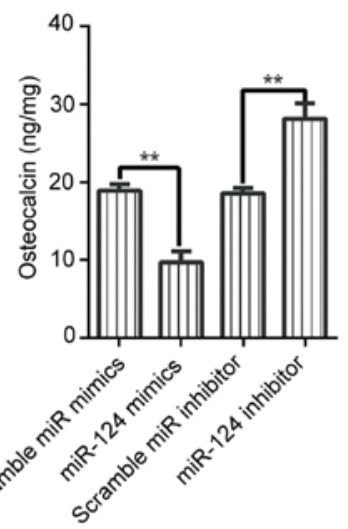

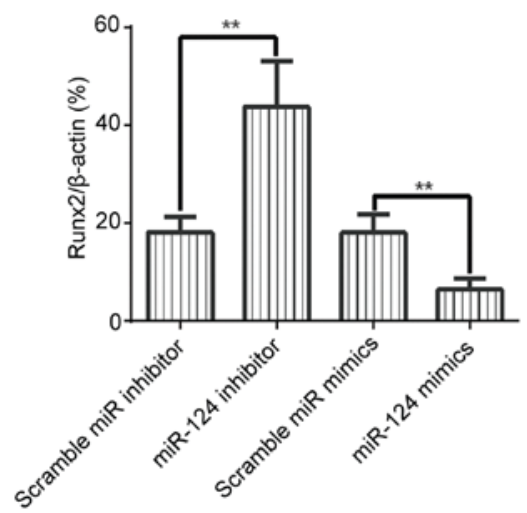

Figure 2. miR-124 regulates osteogenic differentiation of BMSCs. (A) BMSCs were transfected with scramble miR mimics, miR-124 mimics, scramble miR inhibitor, or miR-124 inhibitor. The expression of miR-124 was determined by RT-qPCR. (B-D) BMSCs were transfected and cultured in OM. ALP activity, OC secretion, and the level of Runx 2 were assessed. Data are expressed as mean $\pm \mathrm{SD}, \mathrm{n}=3$. ${ }^{* *} \mathrm{P}<0.01$. BMSCs, bone marrow-derived mesenchymal stem cells; ALP, alkaline phosphatase; OC, osteocalcin; CM, control medium; OM, osteogenic medium. 
A

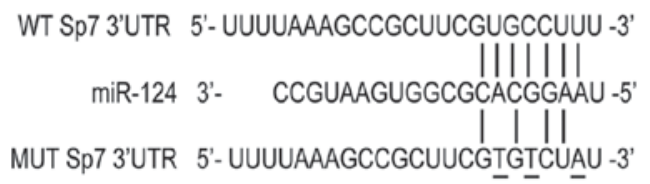

C

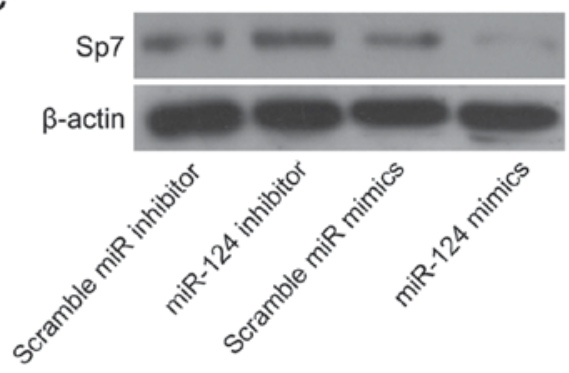

B
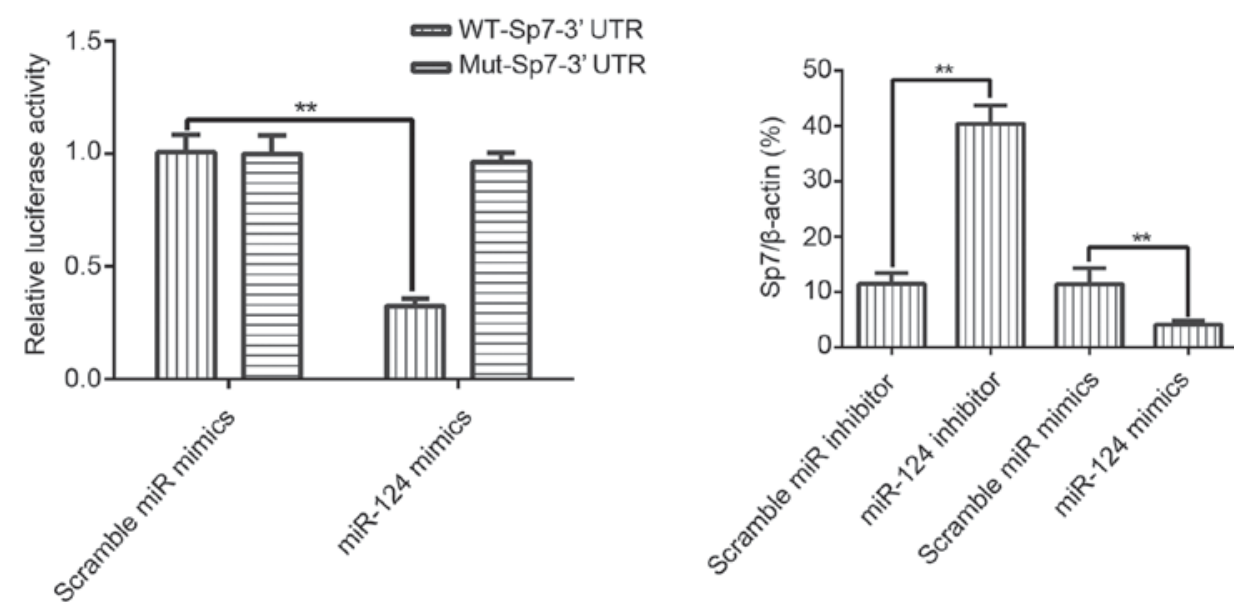

Figure 3. Sp7 is a target of miR-124. (A) Schematic of the miR-124 putative target sites in the Sp7 3'untranslated region (UTR) and alignment of miR-124 with the wild-type (WT) Sp7 3'UTR showing complementary pairing. The three mutated nucleotides are underlined. (B) Luciferase reporter assay was performed using luciferase constructs carrying WT or MUT-Sp7 3'UTR cotransfected with miR-124 mimics or scramble control into BMSCs. (C) BMSCs were transfected with scramble miR inhibitor, miR-124 inhibitor, scramble miR mimics, or miR-124 mimics and the expression of Sp7 was determined by western blotting. Data are expressed as means $\pm \mathrm{SD}, \mathrm{n}=3 .{ }^{* * *} \mathrm{P}<0.01$. BMSCs, bone marrow-derived mesenchymal stem cells; WT, wild-type; MUT, mutant.

cartilage, tendon, and muscle, and thus hold great potential for therapeutic applications $(1,22,23)$. Recent studies have shown that certain miRNAs may play a key role in osteogenic differentiation of BMSCs (24,25). However, the molecular mechanisms governing osteogenic differentiation of BMSCs involved in the pathogenesis of bone homeostasis remain unclear. In the present study, human BMSCs were isolated and cultured from three individual donors. Osteogenic medium was used to induce osteogenic differentiation of BMSCs, which was confirmed by an increase in ALP activity, OC secretion, Runx 2 and Sp7 protein expression, and the formation of mineralized nodules.

miRNAs, important post-transcriptional regulators in gene expression (26), play a pivotal role in the functions of BMSCs. Recently, many miRNAs have been reported to modulate the phenotypes and differentiation of BMSCs (27-29). A previous study demonstrated that miR-144-3p negatively regulated osteogenic differentiation and proliferation of MSCs by specifically targeting Smad4 (27). A study by Jeong et al suggested that miR-194 could determine whether MSCs would differentiate into osteoblasts or adipocytes by targeting COUP-TFII (28). Yang et al showed that TNF- $\alpha$ inhibited osteoblastic differentiation of MSCs by suppressing the functional axis of miR-21 and Spry1 (29). These results indicated that miRNAs play a vital role in regulating the osteogenic differentiation of MSCs.
Recently, miR-124 has received much attention from researchers for its key role in the different differentiation pathways of MSCs. For example, Qadir et al showed that miR-124 acts as a negative regulator of myogenic differentiation of MSCs by targeting Dlx5 (12) and another study showed that miR-124 regulated cardiomyocyte differentiation of BMSCs by targeting STAT3 mRNA (30). In addition, Zou et al demonstrated that miR-124 plays an important role in the differentiation of BMSCs into neurons (13). In the present study, it was also identified that miR-124 was downregulated during osteogenic differentiation of BMSCs and involved in regulating osteogenic differentiation of BMSCs. That is, ALP activity, OC secretion and Runx 2 protein expression were significantly decreased in miR-124-overexpressing BMSCs while inhibition of the expression of miR-124 led to opposite results. These results are consistent with one previous study, which showed that miR-124 is also negatively correlated with osteogenic differentiation of MSCs by targeting Dlx3, Dlx5, and Dlx2 (14). However, the role of miR-124 in the osteogenic differentiation of BMSCs in vivo needs further study.

Sp7, a member of the Sp family of zinc-finger-containing transcription factors, is a bone-specific transcription factor that plays a crucial role in osteoblast mineralization $(31,32)$. Previous studies have shown that BMSCs are multipotent and exhibit different levels of Sp7 expression depending on the 
A
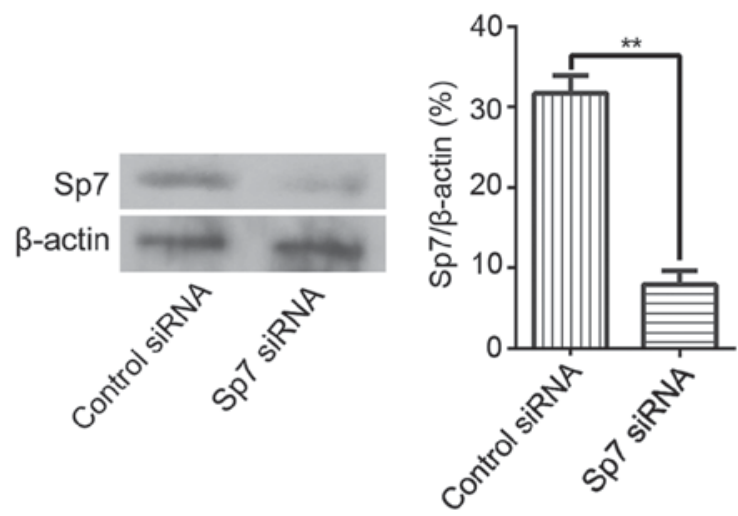

C

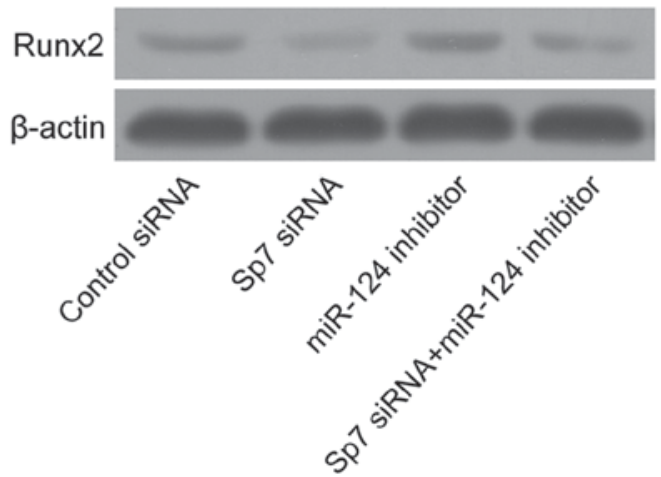

$\mathrm{B}$
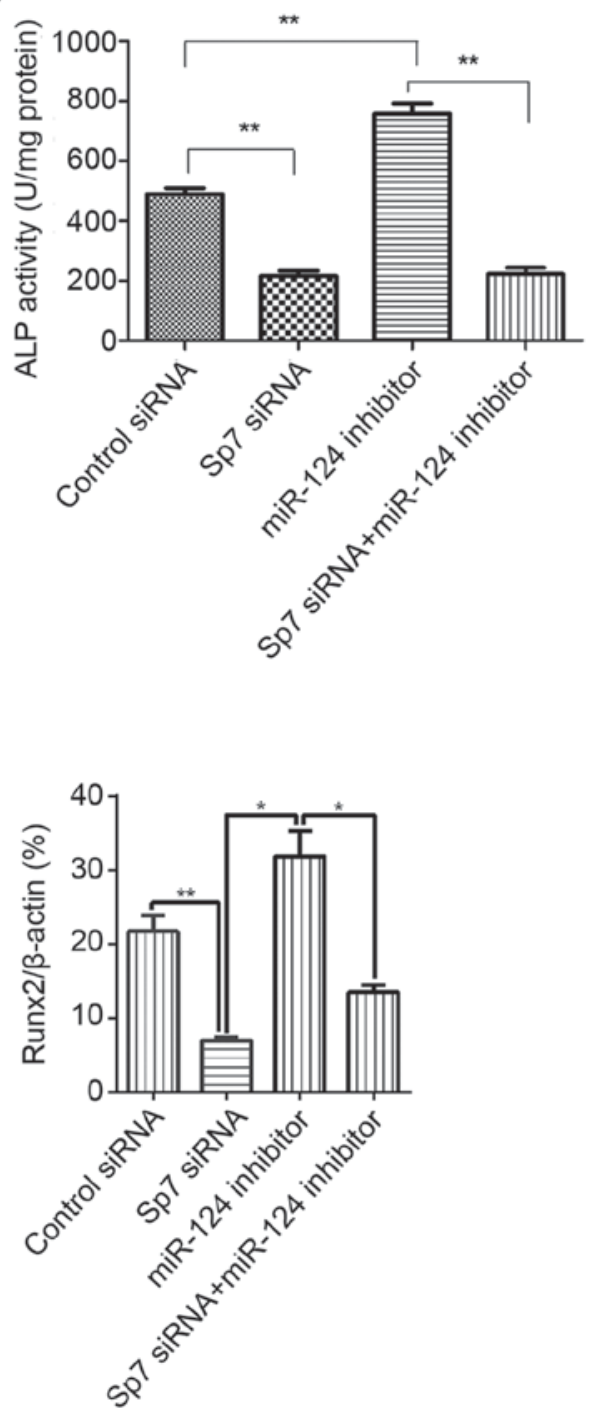

Figure 4. Sp7 is involved in miR-124 regulation of osteogenic differentiation of BMSCs. (A) Expression of Sp7 in BMSCs transfected with Sp7 small interfering RNA (siRNA) or control siRNA was determined by western blotting. (B and C) BMSCs were transfected with control siRNA, Sp7 siRNA, miR-204 inhibitor, or Sp7 siRNA + miR-124 inhibitor and ALP activity and Runx2 protein were determined by spectrophotometry and western blotting, respectively. Data are expressed as means $\pm \mathrm{SD}, \mathrm{n}=3$. ${ }^{*} \mathrm{P}<0.05,{ }^{* * *} \mathrm{P}<0.01$. BMSCs, bone marrow-derived mesenchymal stem cells; ALP, alkaline phosphatase.

differentiation commitment $(33,34)$. Previous studies have also shown that miR-125b regulates the osteogenic differentiation of both BMSCs (11) and VSMCs (35) by targeting Sp7. Yang et al demonstrated that miR-93 regulates osteoblast mineralization through a novel miR-93/Sp7 regulatory feedback loop (32). In the present study, bioinformatic analysis predicted that $\mathrm{Sp} 7$ is a potential target of miR-124 and it was demonstrated that $\mathrm{Sp} 7$ is the direct target of miR-124. On the one hand, miR-124 negatively regulates the expression of Sp7. On the other hand, overexpression of miR-124 significantly suppressed the luciferase activity of the WT-Sp7 3'UTR reporter but not that of the MUT-Sp7 3'UTR reporter. Moreover, knockdown of the expression of Sp7 using siRNA blocked the osteogenic differentiation of BMSCs induced by the miR-124 inhibitor, suggesting that the effect of miR-124 in regulating osteogenic differentiation is dependent on Sp7. Collectively, the data supported that miR-124 attenuates osteogenic differentiation of BMSCs by directly targeting Sp7.
In conclusion, the present study identified for the first time that miR-124 is a significant regulator of osteogenic differentiation of BMSCs by targeting Sp7. This finding provides insight into the mechanisms of osteogenic differentiation of BMSCs and suggests that miR-124 may be an important potential therapeutic target for a variety of bone diseases including osteoporosis.

\section{Acknowledgements}

Not applicable.

\section{Funding}

The present study was supported by funding from the National Natural Science Foundation of China (nos. 81770881 and 81870623), the Fundamental Research Funds for the Central Universities of Central South University (nos. 2018zzts918 and 2 018zzts048) and the National Basic Research Program of China (973 Program) (no. 2014CB942903). 


\section{Availability of data and materials}

The datasets used during the present study are available from the corresponding author upon reasonable request.

\section{Authors' contributions}

LQY and FL conceived and designed the experiments. JZT and XL performed the experiments, analyzed the data and prepared all the figures. JYZ, FX, RRC, FW and XBL provided technical support, made substantial contributions to data analysis and revised the manuscript critically for important intellectual content. JZT and XL wrote the manuscript. All authors read and approved the manuscript and agree to be accountable for all aspects of the research in ensuring that the accuracy or integrity of any part of the work are appropriately investigated and resolved.

\section{Ethics approval and consent to participate}

All of the subjects provided informed consent. Approval for the present study was acquired from the Medical Ethics Committee of The Second Xiang-Ya Hospital, Central South University.

\section{Patient consent for publication}

Not applicable.

\section{Competing interests}

The authors state that they have no competing interests.

\section{References}

1. Pittenger MF, Mackay AM, Beck SC, Jaiswal RK, Douglas R, Mosca JD, Moorman MA, Simonetti DW, Craig S and Marshak DR: Multilineage potential of adult human mesenchymal stem cells. Science 284: 143-147, 1999.

2. Tuli R, Tuli S, Nandi S, Wang ML, Alexander PG, Haleem-Smith H, Hozack WJ, Manner PA, Danielson KG and Tuan RS: Characterization of multipotential mesenchymal progenitor cells derived from human trabecular bone. Stem Cells 21: 681-693, 2003.

3. Rachner TD, Khosla S and Hof bauer LC: Osteoporosis: Now and the future. Lancet 377: 1276-1287, 2011.

4. Nuttall ME and Gimble JM: Controlling the balance between osteoblastogenesis and adipogenesis and the consequent therapeutic implications. Curr Opin Pharmacol 4: 290-294, 2004.

5. Cui RR, Li SJ, Liu LJ, Yi L, Liang QH, Zhu X, Liu GY, Liu Y, Wu SS, Liao XB, et al: MicroRNA-204 regulates vascular smooth muscle cell calcification in vitro and in vivo. Cardiovasc Res 96: 320-329, 2012.

6. Lin X, Xu F, Cui RR, Xiong D, Zhong JY, Zhu T, Li F, Wu F, $\mathrm{Xie} \mathrm{XB}$, Mao MZ, et al: The arterial calcification is regulated via a miR-204/DNMT3a regulatory circuit both in vitro and in female mice. Endocrinology 159: 2905-2916, 2018.

7. Liao XB, Zhang ZY, Yuan K, Liu Y, Feng X, Cui RR, $\mathrm{Hu}$ YR, Yuan ZS, Gu L, Li SJ, et al: MiR-133a modulates osteogenic differentiation of vascular smooth muscle cells. Endocrinology 154: 3344-3352, 2013.

8. Liu Y, Xu F, Pei HX, Zhu X, Lin X, Song CY, Liang QH, Liao EY and Yuan LQ: Vaspin regulates the osteogenic differentiation of MC3T3-E1 through the PI3K-Akt/miR-34c loop. Sci Rep 6: 25578, 2016.

9. Liu Y, Zhang XL, Chen L, Lin X, Xiong D, Xu F, Yuan LQ and Liao EY: Epigenetic mechanisms of bone regeneration and homeostasis. Prog Biophys Mol Biol 122: 85-92, 2016.
10. Li Z, Hassan MQ, Volinia S, van Wijnen AJ, Stein JL, Croce CM, Lian JB and Stein GS: A microRNA signature for a BMP2-induced osteoblast lineage commitment program. Proc Natl Acad Sci USA 105: 13906-13911, 2008.

11. Chen S, Yang L, Jie Q, Lin YS, Meng GL, Fan JZ, Zhang JK, Fan J, Luo ZJ and Liu J: MicroRNA125b suppresses the proliferation and osteogenic differentiation of human bone marrowderived mesenchymal stem cells. Mol Med Rep 9: 1820-1826, 2014.

12. Qadir AS, Woo KM, Ryoo HM, Yi T, Song SU and Baek JH: MiR-124 inhibits myogenic differentiation of mesenchymal stem cells via targeting Dlx5. J Cell Biochem 115: 1572-1581, 2014.

13. Zou D, Chen Y, Han Y, Lv C and Tu G: Overexpression of microRNA-124 promotes the neuronal differentiation of bone marrow-derived mesenchymal stem cells. Neural Regen Res 9: 1241-1248, 2014

14. Qadir AS, Um S, Lee H, Baek K, Seo BM, Lee G, Kim GS, Woo KM, Ryoo HM and Baek JH: miR-124 negatively regulates osteogenic differentiation and in vivo bone formation of mesenchymal stem cells. J Cell Biochem 116: 730-742, 2015.

15. Liang QH, Jiang Y, Zhu X, Cui RR, Liu GY, Liu Y, Wu SS, Liao XB, Xie H, Zhou HD, et al: Ghrelin attenuates the osteoblastic differentiation of vascular smooth muscle cells through the ERK pathway. PLoS One 7: e33126, 2012.

16. Zhang W, Guo H, Jing H, Li Y, Wang X, Zhang H, Jiang L and Ren F: Lactoferrin stimulates osteoblast differentiation through PKA and p38 pathways independent of lactoferrin's receptor LRP1. J Bone Miner Res 29: 1232-1243, 2014.

17. Yuan L, Chan GC, Fung KL and Chim CS: RANKL expression in myeloma cells is regulated by a network involving RANKL promoter methylation, DNMT1, microRNA and TNF $\alpha$ in the microenvironment. Biochim Biophys Acta 1843: 1834-1838, 2014.

18. Liu GY, Liang QH, Cui RR, Liu Y, Wu SS, Shan PF, Yuan LQ and Liao EY: Leptin promotes the osteoblastic differentiation of vascular smooth muscle cells from female mice by increasing RANKL expression. Endocrinology 155: 558-567, 2014.

19. Livak KJ and Schmittgen TD: Analysis of relative gene expression data using real-time quantitative PCR and the 2(-Delta Delta C(T)) method. Methods 25: 402-408, 2001.

20. Peng YQ, Xiong D, Lin X, Cui RR, Xu F, Zhong JY, Zhu T, Wu F, Mao MZ, Liao XB and Yuan LQ: Oestrogen inhibits arterial calcification by promoting autophagy. Sci Rep 7: 3549, 2017.

21. Wu SS, Liang QH, Liu Y, Cui RR, Yuan LQ and Liao EY: Omentin-1 stimulates human osteoblast proliferation through PI3K/Akt signal pathway. Int J Endocrinol 2013: 368970, 2013.

22. Qian J, Hu Y, Zhao L, Xia J, Li C, Shi L and Xu F: Protective role of adipose-derived stem cells in staphylococcus aureus-induced lung injury is mediated by RegIII $\gamma$ secretion. Stem Cells 34: 1947-1956, 2016.

23. Xu F, Hu Y, Zhou J and Wang X: Mesenchymal stem cells in acute lung injury: Are they ready for translational medicine? J Cell Mol Med 17: 927-935, 2013.

24. Eskildsen T, Taipaleenmäki H, Stenvang J, Abdallah BM, Ditzel N, Nossent AY, Bak M, Kauppinen S and Kassem M: MicroRNA-138 regulates osteogenic differentiation of human stromal (mesenchymal) stem cells in vivo. Proc Natl Acad Sci USA 108: 6139-6144, 2011.

25. Kang $\mathrm{H}$ and Hata A: The role of microRNAs in cell fate determination of mesenchymal stem cells: Balancing adipogenesis and osteogenesis. BMB Rep 48: 319-323, 2015.

26. Ying H, Kang Y, Zhang H, Zhao D, Xia J, Lu Z, Wang H, Xu F and Shi L: MiR-127 modulates macrophage polarization and promotes lung inflammation and injury by activating the JNK pathway. J Immunol 194: 1239-1251, 2015.

27. Huang C, Geng J, Wei X, Zhang R and Jiang S: MiR-144-3p regulates osteogenic differentiation and proliferation of murine mesenchymal stem cells by specifically targeting Smad4. FEBS Lett 590: 795-807, 2016.

28. Jeong BC, Kang IH, Hwang YC, Kim SH and Koh JT: MicroRNA-194 reciprocally stimulates osteogenesis and inhibits adipogenesis via regulating COUP-TFII expression. Cell Death Dis 5: e1532, 2014.

29. Yang N, Wang G, Hu C, Shi Y, Liao L, Shi S, Cai Y, Cheng S, Wang X, Liu Y, et al: Tumor necrosis factor $\alpha$ suppresses the mesenchymal stem cell osteogenesis promoter miR-21 in estrogen deficiency-induced osteoporosis. J Bone Miner Res 28: 559-573, 2013.

30. Cai B, Li J, Wang J, Luo X, Ai J, Liu Y, Wang N, Liang H, Zhang M, Chen N, et al: microRNA-124 regulates cardiomyocyte differentiation of bone marrow-derived mesenchymal stem cells via targeting STAT3 signaling. Stem Cells 30: 1746-1755, 2012. 
31. Nakashima K, Zhou X, Kunkel G, Zhang Z, Deng JM, Behringer RR and de Crombrugghe B: The novel zinc finger-containing transcription factor osterix is required for osteoblast differentiation and bone formation. Cell 108: 17-29, 2002.

32. Yang L, Cheng P, Chen C, He HB, Xie GQ, Zhou HD, Xie H, Wu XP and Luo XH: miR-93/Sp7 function loop mediates osteoblast mineralization. J Bone Miner Res 27: 1598-1606, 2012.

33. Tu Q, Valverde P and Chen J: Osterix enhances proliferation and osteogenic potential of bone marrow stromal cells. Biochem Biophys Res Commun 341: 1257-1265, 2006.
34. Zhou X, Zhang Z, Feng JQ, Dusevich VM, Sinha K, Zhang H, Darnay BG and de Crombrugghe B: Multiple functions of Osterix are required for bone growth and homeostasis in postnatal mice. Proc Natl Acad Sci USA 107: 12919-12924, 2010.

35. Goettsch C, Rauner M, Pacyna N, Hempel U, Bornstein SR and Hofbauer LC: miR-125b regulates calcification of vascular smooth muscle cells. Am J Pathol 179: 1594-1600, 2011. 